\title{
Reply to J. Heinrich
}

To the Editor,

We agree with J. Heinrich that randomised controlled trials are the gold standard to infer causality ${ }^{1}$. According to the World Cancer Research Foundation ${ }^{2}$, results from observational studies can however be used to support evidence for causal associations. This is even more valuable that randomized control trials are not always feasible or ethical, as noted by J. Heinrich. Noteworthy, breastfeeding benefits evidence was mostly based on observational studies ${ }^{3}$.

A strength of prospective studies is the ability to examine the association between consumption of a specific product and health in real conditions of use. Firstly, in the national Elfe cohort, we observed that a large part of infants with no familial history of allergy consumed partially hydrolysed formula with hypoallergenic label (pHF-HA) (24\%, according to Supplementary table 2 in our published paper ${ }^{4}$ ), whereas current guidelines encourage the use of pHF only for infants with familial history of allergy. According to a recent systematic review and expert consensus ${ }^{5}$, data are insufficient to support potential benefit of pHF on allergy prevention in not-at-risk infants. It is therefore of great importance to assess health effects of these formulas also among non-at-risk infants. Even in at-risk infants, the beneficial effects of $\mathrm{pHF}$ formula remains inconsistent ${ }^{5,6}$. In particular, although the health claim allowed for these formulas is about reducing the risk of allergy to milk proteins, no study has reported a beneficial effect on cow's milk protein allergy, neither on other food allergies.

Whereas we acknowledge that a major issue of observational studies is to address potential confounding and reverse causation bias, this was thoroughly discussed in our manuscript, in light of different models performed. In fact, we addressed the reverse causation bias (i.e. pHF formula prescribed to more at-risk infants or to infants with suspected allergic symptoms) by excluding all infants with cow's milk protein allergy reported at the 2-month follow-up from our analyses and stratifying all analyses on familial history of allergy. In a second step, we also excluded all infants with eczema, wheezing or gastroesophageal reflux reported at the 2-month follow-up from our analyses. We acknowledged that for eczema and food allergy in the at-risk subgroup, the association with the use of pHF-HA was no longer significant after these exclusions, thus suggesting that their higher risk of subsequent allergies was related to these early symptoms. However, our analysis brings no argument to suggest that in these infants with eczema, wheezing or gastroesophageal reflux at 2 months, feeding with HA formula would mitigate their subsequent risk of allergic manifestations at 1 and 2 years. Moreover, the observed association was still significant for food allergy in the non-at-risk subgroup and for wheezing at 1 year in the at-risk group. Not to mention that the association with pHF-HA became significant for wheezing and asthma attack at 2 
years, in the at-risk and the non-at-risk subgroups, respectively. As reported in previous reviews ${ }^{5}$, pHFs may differ according to the source proteins and then should not be considered equivalent. In our paper ${ }^{4}$, we first considered all pHFs together in our main analyses and then, to address this issue, we conducted a sensitivity analysis considering only whey-based pHFs, leading to similar findings (supplementary tables 4 and 5), but were not able to account for the hydrolysis level.

We considered that all our sensitivity analyses did not strongly modify our conclusion, that we do not highlight any beneficial effects of pHF-HA formula on allergic symptoms prevention in childhood, and even suspected a potentially harmful effect. However, as this study is the first to report such an association, we underlined the need to conduct further analyses to confirm or infirm our findings, in order to know if the guidelines need to be revised.

Camille Davisse-Paturet ${ }^{1}$, Chantal Raherison ${ }^{2}$, Karine Adel-Patient ${ }^{3}$, Amandine DivaretChauveau $^{4,5}$, Marie-Noëlle Dufourg ${ }^{6}$, Sandrine Lioret ${ }^{1}$, Marie-Aline Charles ${ }^{1,6}$, Blandine de Lauzon-Guillain ${ }^{1}$

${ }^{1}$ Université de Paris, CRESS, INSERM, INRA, Paris, France; ${ }^{2}$ Bordeaux University, Inserm, Bordeaux Population Health Research Center, Team EPICENE, UMR 1219, Bordeaux, France; ${ }^{3}$ UMR Service de Pharmacologie et Immunoanalyse, CEA, INRA, Université Paris-Saclay, Gif-surYvette, France; ${ }^{4}$ Unité d'allergologie pédiatrique, Hôpital d'enfants, CHRU de Nancy, France; 5 EA3450, Université de Lorraine, Vandoeuvre les Nancy, France; ${ }^{6}$ Ined, Inserm, Joint Unit Elfe, Paris, France.

blandine.delauzon@inserm.fr

\section{References}

1. Heinrich J. Comment on recent article by Davisse-Paturet et al. Pediatr Allergy Immunol. 2019.

2. World Cancer Research Fund / American Institute for Cancer Research. Coutinuous Update Project Expert Report 2018. Judging the evidence. Available at dietandcancerreport.org2018.

3. Victora CG, Bahl R, Barros AJ, et al. Breastfeeding in the 21st century: epidemiology, mechanisms, and lifelong effect. Lancet. 2016;387(10017):475-490.

4. Davisse-Paturet C, Raherison C, Adel-Patient K, et al. Use of partially hydrolysed formula in infancy and incidence of eczema, respiratory symptoms or food allergies in toddlers from the ELFE cohort. Pediatr Allergy Immunol. 2019;30(6):614-623. 
5. Vandenplas Y, Latiff AHA, Fleischer DM, et al. Partially hydrolyzed formula in nonexclusively breastfed infants: A systematic review and expert consensus. Nutrition. 2019;57:268-274.

6. Osborn DA, Sinn JK, Jones LJ. Infant formulas containing hydrolysed protein for prevention of allergic disease. Cochrane Database Syst Rev. 2018;10:CD003664. 